\title{
Energy-Efficient Soft-Assisted Product Decoders
}

\author{
Christoffer Fougstedt $^{1}$, Alireza Sheikh ${ }^{2}$, Alexandre Graell i Amat ${ }^{2}$, Gianluigi Liva $^{3}$, \\ and Per Larsson-Edefors ${ }^{1}$ \\ ${ }^{1}$ Dept. of Computer Science and Engineering, ${ }^{2}$ Dept. of Electrical Engineering, Chalmers University of Technology, Sweden \\ ${ }^{3}$ Institute of Communications and Navigation, German Aerospace Center (DLR), Germany \\ chrfou@chalmers.se
}

\begin{abstract}
We implement a 1-Tb/s 0.63-pJ/bit soft-assisted product decoder in a 28-nm technology. The decoder uses one bit of soft information to improve its net coding gain by $0.2 \mathrm{~dB}$, reaching 10.3-10.4 dB, which is similar to that of more complex hard-decision staircase decoders.
\end{abstract}

OCIS codes: (060.0060) Fiber optics and optical communication; (060.2330) Fiber optics communications

\section{Introduction}

Forward error correction (FEC) codes decoded using hard-decision (HD) decoding, such as product and staircase codes, are commonly considered for moderate-reach high-throughput fiber-optic communication systems as they offer a relatively high net coding gain (NCG) while using low-complexity decoders. But often there is soft information available in DSP-based fiber-optic receivers; soft information that can be harnessed to improve decoding performance. In contrast to soft-decision (SD) decoding, which typically operates using fixed-point math and entails a significant cost in implementation complexity for operations, data storage, and update requirements, hybrid HD-SD decoding schemes, such as binary message-passing low-density parity-check (LDPC) decoders operating on quantized channel information [1] and recently proposed low-complexity decoding algorithms for product codes [2,3], can allow a decoder to operate internally on hard decisions, but selectively use soft information during decoding to significantly improve the NCG. With the rationale that the NCG can be improved at a very minor increase in implementation complexity over HD decoders, we propose and evaluate a VLSI architecture of a soft-assisted product decoder based on the iBDD-SR algorithm [2,3], with a decoder core based on our earlier staircase decoder architecture [4].

The proposed soft-assisted product decoder uses a single bit of soft information. Remarkably, this allows us to achieve similar coding gains as more complex staircase decoders $[4,5]$. While staircase decoders provide high NCG using pure HD decoding, they require a large data-processing memory due to the windowed decoding operation. In contrast, the proposed iBDD-SR soft-assisted product decoder has lower circuit complexity, leading to less circuit area and decoding latency. In addition, since the decoder core uses hard decisions only, it can support significantly higher throughputs than SD decoders [6], making our proposed iBDD-SR soft-assisted product decoder a viable option for very-high-throughput high-NCG systems.

\section{Decoder Algorithm and Architecture}

Product codes can provide high NCG when employing low-complexity iterative bounded-distance decoding (iBDD) [7]. In iBDD, bounded distance decoding of the component codes is performed in an iterative row/column manner. However, there is a probability that the component decoders introduce miscorrections, which reduce the achievable NCG. Recently, iBDD-SR was proposed [2], in which the channel soft information is used to reduce the component-decoder miscorrection rate. Here, we briefly review the iBDD-SR algorithm and show that we can simplify the algorithm to the addition of one memory element and one logic gate per bit, with respect to the underlying iBDD.

Let us consider the decoding of the $i$ th row component code, in particular, the result of iBDD-SR corresponding to code bit $c_{i, j}$. Let $\bar{\mu}_{i, j}^{\mathrm{r},(\ell)} \in\{ \pm 1,0\}$ be the result of iBDD on code bit $c_{i, j}$ in iteration $\ell$, where 0 represents a decoding failure. In iBDD-SR, the message sent to the $j$ th column codes through $c_{i, j}$ is computed as $\psi_{i, j}^{\mathrm{r},(\ell)}=\mathrm{B}\left(w_{\ell} \cdot \bar{\mu}_{i, j}^{\mathrm{r},(\ell)}+L_{i, j}\right)$, where $w_{\ell}>0$ is a scaling parameter that can be optimized to reduce the bit error rate (BER), $L_{i, j}$ is the reliability of $c_{i, j}$, and $\mathrm{B}(\cdot)$ takes the sign of the input and maps $-1 \mapsto 1$ and $+1 \mapsto 0$ (see [2] for details).

The range of $w_{\ell}$ is small and can thus be fixed to a single value at the expense of a minor degradation in BER performance [2]. In this case, $\psi_{i, j}^{\mathrm{r},(\ell)}=\mathrm{B}\left(w \cdot \bar{\mu}_{i, j}^{\mathrm{r},(\ell)}+L_{i, j}\right)$ can be simplified to a comparison that returns $\mathrm{B}\left(\bar{\mu}_{i, j}^{\mathrm{r},(\ell)}\right)$ if $L_{i, j}<w$ and $\mathrm{B}\left(L_{i, j}\right)$ otherwise. Thus, we need only to store whether a certain received bit has a lower reliability than $w$ and only apply corrections for those bits, while rejecting attempts to flip high-reliability bits; as we will show, this can be implemented using low-complexity circuits. After performing a given number of iterations using iBDD-SR, we 


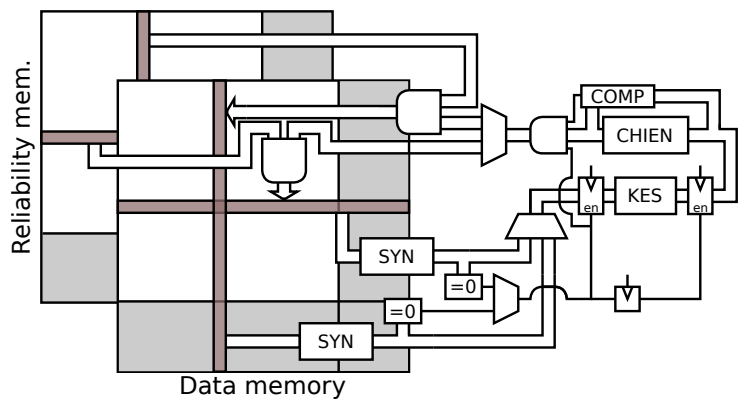

(a) Block diagram of the soft-assisted product decoder.

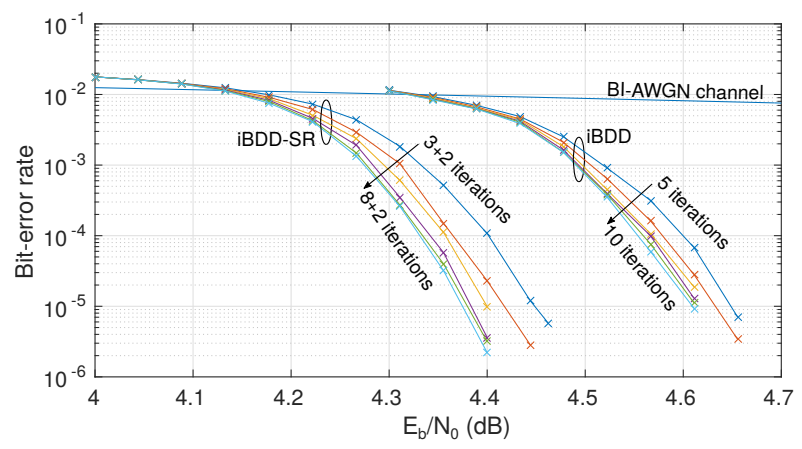

(b) Output BER as a function of $E_{\mathrm{b}} / N_{0}$.

Fig. 1: Decoder circuit architecture and BER performance.

perform two iBDD iterations to correct erroneous bits mistakenly labeled as reliable and thus not corrected during the iBDD-SR iterations, cleaning up the few remaining errors.

We consider transmission over a binary-input additive white Gaussian noise (BI-AWGN) channel, and a fixed scaling parameter of 0.587 . The output of the channel is quantized (without $E_{\mathrm{b}} / N_{0}$ normalization) to two bits: one HD bit and one reliability bit. The decoder stores the channel output in two memories: one reliability memory, which is only updated once a new block is received, and one data memory, on which the component decoders iteratively operate.

\section{VLSI Decoder Architecture}

We consider a product code with $\mathrm{BCH}(255,231,3)$ component codes giving an overall overhead of $21.9 \%$ and a block length of 65,025 bits. The HD product decoder core, which shares architectural features with the staircase decoder in [4], employs fast and non-iterative component decoders. This allows for very high throughputs, while still keeping the vast majority of the logic circuits static over row/column iterations, resulting in very low energy dissipation. In addition to the HD bit, one reliability bit has to be stored. The reliability bit is used to mask corrections to bits which are considered reliable. This reduces the miscorrection rate, while increasing the complexity by one memory element and one AND-gate per bit.

Fig. 1a shows a block diagram of the implemented decoder. The addition of circuits to handle reliability information increases the total circuit area by a mere 5\%. Since component decoders perform correction relatively sporadically, we can employ extensive clock gating to reduce the overall power dissipation. The reliability memory is updated on the arrival of a new data block and kept idle otherwise. Replicated syndrome-computation units are employed, allowing us to keep their inputs largely static during iterations and thus decreasing power dissipation at a small increase in area. The decoder processing memory is only clocked at the input of a new block and when a component decoder applies a correction. The component decoder pipelines are sequentially gated if a zero-syndrome is found.

\subsection{Decoder Performance Evaluation}

The hardware description (VHDL) implementations of the soft-assisted (iBDD-SR) and HD (iBDD) decoders were simulated using a VHDL-based quantized-output BI-AWGN channel. By simulating the actual VHDL implementation, we verify the actual decoder BER performance. Fig. $1 \mathrm{~b}$ shows the output BER as a function of $E_{\mathrm{b}} / N_{0}$. Simulation runtime limits low-BER statistics and we need to resort to extrapolation to estimate the NCG at an output BER of $10^{-15}$. Here, we use berfit in MATLAB to extrapolate to $10^{-15}$. Since low-BER statistics are limited, we remark that the extrapolated results should be seen as approximate NCG. We estimate the NCG of iBDD-SR to 10.3-10.4 dB and 10.1-10.2 dB for iBDD, as we vary the iteration count from 5 to 10 . Thus, using a single bit of soft information allows us to approach the NCGs achieved by staircase codes at similar code rates $[4,5]$.

\subsection{Circuit Implementation and Evaluation}

The decoders were synthesized using Cadence Genus and a 0.9-V 28-nm fully-depleted silicon-on-insulator CMOS standard-cell flow, with regular threshold voltage cells, characterized at the slow process corner at $125^{\circ} \mathrm{C}$, using physical wire models and a clock rate of $600 \mathrm{MHz}$. The implemented decoders were simulated using uniformly-distributed encoded data transmitted over the implemented BI-AWGN channel model in order to generate internal switching activity statistics for accurate power dissipation estimation. The switching activity was then back-annotated to the netlist in Cadence Genus, and power dissipation estimation was performed at the typical process corner and $25^{\circ} \mathrm{C}$. 
Table 1: VLSI Implementation Evaluation Results

\begin{tabular}{|c|c|c|c|c|c|c|c|c|c|c|c|c|}
\hline & \multicolumn{6}{|c|}{ Soft-Assisted Decoder (iBDD-SR) } & \multicolumn{6}{|c|}{ Hard-Decision Decoder (iBDD) } \\
\hline $\begin{array}{l}\text { Estimated net coding gain } \\
\text { Cell area and gate count }\end{array}$ & \multicolumn{6}{|c|}{$\begin{array}{c}10.3 \mathrm{~dB}-10.4 \mathrm{~dB} \\
4.76 \mathrm{~mm}^{2}, 9722 \mathrm{kGates}\end{array}$} & \multicolumn{6}{|c|}{$\begin{array}{c}10.1 \mathrm{~dB}-10.2 \mathrm{~dB} \\
4.52 \mathrm{~mm}^{2}, 9232 \mathrm{kGates}\end{array}$} \\
\hline Iterations & 5 & 6 & 7 & 8 & 9 & 10 & 5 & 6 & 7 & 8 & 9 & 10 \\
\hline Information through & 1000 & 842 & 728 & 640 & 572 & 516 & 1000 & 842 & 728 & 640 & 572 & 516 \\
\hline Power dissipation (mW) @ $5.2 \mathrm{~dB}$ & 633 & 591 & 561 & 534 & 516 & 502 & 508 & 447 & 403 & 366 & 340 & 321 \\
\hline Energy/info. bit (pJ/bit) @5.2 dB & 0.63 & 0.70 & 0.77 & 0.83 & 0.90 & 0.97 & 0.51 & 0.53 & 0.55 & 0.57 & 0.59 & 0.62 \\
\hline Energy/info. bit (pJ/bit) @threshold & 0.81 & 0.88 & 0.95 & 1.01 & 1.08 & 1.16 & 0.61 & 0.63 & 0.65 & 0.66 & 0.69 & 0.72 \\
\hline Block decoding latency (ns) & 53 & 63 & 73 & 83 & 93 & 103 & 53 & 63 & 73 & 83 & 93 & 103 \\
\hline
\end{tabular}

\section{Results and Discussion}

Table 1 shows the evaluation results of the implemented product decoders. Decoder energy dissipation depends on $E_{\mathrm{b}} / N_{0}$ and, thus, the decoders are evaluated both at $E_{\mathrm{b}} / N_{0}=5.2 \mathrm{~dB}$ (corresponding to an input BER of $10^{-2}$ ), and at the approximate $10^{-15}$ threshold (4.6 and $4.9 \mathrm{~dB}$ for $\mathrm{iBDD}-\mathrm{SR}$ and $\mathrm{iBDD}$, respectively). Since the HD core is common for both implementations, both the soft-assisted and the HD decoder achieve up to $1 \mathrm{~Tb} / \mathrm{s}$ of information throughput. However, the soft-assisted decoder achieves $0.2 \mathrm{~dB}$ higher NCG at the expense of just $5 \%$ increase in area and gate count (defined as cell area normalized to the smallest 2-input NAND gate available in the standard-cell library), and $0.12-0.30 \mathrm{pJ} / \mathrm{bit}$ increase in energy dissipation. While the power dissipation decreases as more iterations are performed, the number of errors (and thus required component-code corrections) progressively reduces, and the energy per information bit increases since the decoder throughput is reduced. The decoders achieve a block-decoding latency of 53-103 ns, depending on the number of decoding iterations performed.

Decoder circuit implementations that achieve a throughput in excess of $500 \mathrm{~Gb} / \mathrm{s}$ are rarely published in the open literature. Compared to a recently published high-throughput SD LDPC decoder [8], our soft-assisted decoder achieves $70 \%$ higher throughput at less than half of the area and a 97\% reduction in power dissipation, all while achieving a higher NCG $\left(E_{\mathrm{b}} / N_{0}=4.5 \mathrm{~dB}\right.$ for a BER of $10^{-7}$, compared to $4.97 \mathrm{~dB}$ in [8]). It should be noted that the implementation in [8] uses a short block-length $\operatorname{LDPC}(2048,1723)$ code. In comparison to our earlier HD staircase decoders [4], the soft-assisted decoder achieves $1 \mathrm{~Tb} / \mathrm{s}$ throughput with an area and energy dissipation which is less than half of the HD staircase decoders, at similar estimated NCGs.

\section{Conclusion}

We presented a soft-assisted product decoder implemented in a 28-nm process technology. The implemented iBDD-SR decoder relies on a high-throughput hard-decision decoder VLSI architecture and can achieve a throughput of $1 \mathrm{~Tb} / \mathrm{s}$, while dissipating $0.63 \mathrm{pJ} /$ information bit. A coding gain of $10.3-10.4 \mathrm{~dB}$ is achieved by exploiting one added softinformation bit and a $21.9 \%$-overhead code. Using soft information, if available, allows us to achieve similar coding gains as HD staircase decoders, with significantly lower circuit area and energy dissipation.

Acknowledgement: This work was financially supported by the Knut and Alice Wallenberg Foundation.

\section{References}

[1] G. Lechner, T. Pedersen, and G. Kramer, "Analysis and design of binary message passing decoders," IEEE Trans. Commun. 60, 601-607 (2012).

[2] A. Sheikh, A. Graell i Amat, and G. Liva, "Iterative bounded distance decoding of product codes with scaled reliability," in "Eur. Conf. Opt. Commun. (ECOC)," (2018).

[3] A. Sheikh, A. Graell i Amat, G. Liva, C. Häger, and H. D. Pfister, "On low-complexity decoding of product codes for high-throughput fiber-optic systems," in "Int. Symp. on Turbo Codes \& Iterative Inf. Proc. (ISTC)," (2018).

[4] C. Fougstedt and P. Larsson-Edefors, "Energy-efficient high-throughput staircase decoders," in "Opt. Fiber Commun. Conf. (OFC)," (2018).

[5] L. M. Zhang and F. R. Kschischang, "Staircase codes with 6\% to 33\% overhead," IEEE J. Lightw. Technol. 32, 1999-2002 (2014).

[6] P. Larsson-Edefors, C. Fougstedt, and K. Cushon, "Implementation challenges for energy-efficient error correction in optical communication systems," in "Advanced Photonics 2018 (SPPCom)," (2018).

[7] J. Justesen, "Performance of product codes and related structures with iterated decoding," IEEE Trans. Commun. 59, 407-415 (2011).

[8] R. Ghanaatian, A. Balatsoukas-Stimming, T. C. Müller, M. Meidlinger, G. Matz, A. Teman, and A. Burg, "A 588-Gb/s LDPC decoder based on finite-alphabet message passing,” IEEE Trans. Very Large Scale Integr. (VLSI) Syst. 26, 329-340 (2018). 